\title{
Control of a multigroup COVID-19 model with immunity: treatment and test elimination
}

\author{
Shidong Zhai • Hui Gao • Guoqiang Luo • \\ Junli Tao 1
}

Received: 27 April 2020 / Accepted: 14 September 2020 / Published online: 30 September 2020

(C) Springer Nature B.V. 2020

\begin{abstract}
This paper introduces a multigroup COVID-19 model with immunity, in which the total population of each group is partitioned into five compartments, that is, susceptible, exposed, infective, infective in treatment and recovered compartment. If the basic reproduction number is less than or equal to one, and the infection graph is strongly connected, then the disease-free equilibrium is globally asymptotically stable and the disease dies out. However, the COVID-19 is already in a pandemic state, and the basic reproduction number is large than one. Hence, in order to make the COVID-19 die out in some groups in an area, we design some appropriate control strategies which reduce the number of exposed people and increase the number of people treated. These two methods have been proved to be the most effective methods at present. An effective algorithm is proposed to identify the groups that need to be controlled. Finally, we use the actual limited data of Hubei, Guangdong and Zhejiang provinces in China to illustrate the effectiveness of the obtained results.
\end{abstract}

\footnotetext{
S. Zhai $(\bowtie) \cdot$ H. Gao · G. Luo

The School of Automation, Chongqing University of Posts and Telecommunications, Chongqing 400065, China

e-mail: zhaisd@cqupt.edu.cn

J. Tao ( $\square)$

Chongqing University Cancer Hospital, Chongqing

400030, China

e-mail: taojunli0712@163.com
}

Keywords Multigroup model - Basic reproduction number $\cdot$ Stability

\section{Introduction}

In December 31, 2019, the Health Commission of Hubei province, China announced that a new coronavirus disease was found in Wuhan, the capital of Hubei province [1]. This coronavirus disease quickly spread around the world, and was tentatively named by World Health Organization (WHO) as the 2019 novel coronavirus (COVID-19) [2,3]. As of July 10, 2020, over 12.27 million people have infected worldwide. Hence, the COVID-19 is now in a state of pandemic worldwide. However, in some local areas, this virus has been completely controlled, such as Hubei, Guangdong and Zhejiang provinces in China. This shows that through strict nucleic acid detection and treatment measures, this virus can be completely controlled in local areas.

The aim of this paper is to introduce a new multigroup COVID-19 model to simulate the outbreak in different groups (country, city or community), and design some appropriate control strategies such that the COVID-19 dies out. To this end, we will first recall the references about multigroup epidemic model. Multigroup epidemic model has been attracting much interest from researchers in various fields because the transmission of many infectious diseases can be modeled in this form, such as mumps, gonorrhea, measles, HIV/AIDS. In reality, the group can describe country, 
city or community. The study of multigroup epidemic model can be traced back to the reference [4], in which a class of SIS multigroup model has been presented. Various multigroup epidemic models have been investigated since then, such as [5-10]. For each multigroup epidemic model, the first task is to study the stability of disease-free equilibrium and endemic equilibrium. If the disease-free equilibrium is globally asymptotically stable, then it means that the disease will eventually die out. However, if the endemic equilibrium is globally asymptotically stable, then it means that the disease will eventually become a pandemic. When the basic reproduction number is less than or equal to one, the diseasefree equilibrium is globally asymptotically stable [8,9]. The stability of endemic equilibrium is more complex, and various methods are presented for dealing with it, such as Lyapunov function [4] and graph-theoretic approach [8]. The above-mentioned multigroup epidemic models generally do not take into account the effects of immunity. For an infectious disease, if there exists an effective vaccine, then this infectious disease can be effectively controlled by expanding the proportion of vaccinated people [11-15]. The treatment and vaccination are two effective ways to control the spread of virus [16,17]. For multigroup epidemic model, there were some references which studied the impact of vaccination, such as multigroup SVEIR epidemic model [18], and multigroup SVIR model with age of vaccination $[18,19]$.

Recently, there have been some studies on multigroup COVID-19 model [20-22]. In [20], the authors introduced a multi-group SEIRA model for the spread of COVID-19, and gave a numerical simulation for this model. In order to sustain the point that social distancing is not enough to control the outbreak of COVID19, a simple multi-group SEIR model was presented in [21]. A multi-group SIR model was presented in [22], including some parameters for presenting different hospitalization and fatality rates among different age groups. Nevertheless, the above different multigroup COVID-19 models do not consider the effective control strategies for the spread of COVID-19, such as strict nucleic acid detection and treatment measures.

In this paper, we propose a new multigroup COVID19 model with immunity. For each group, the total population divides into five compartments, that is, a susceptible compartment, an exposed compartment, an infective compartment, an infective compartment in treatment and a recovered compartment. Assume that part of the population entering a group already has antibodies. Our model can be seen as a generalization of the model in $[12,13]$, and consider the cross infection among different groups. Although vaccination is a very useful method to control the infectious diseases, there are some epidemic viruses that have no vaccine, such as SARS and COVID-19 [1-3,23]. Moreover, it is unrealistic for people to get group immunity naturally. Hence, for an infectious disease without a vaccine, in order to effectively control the spread of this disease, large-scale testing and treatment may be effective methods. For example, for COVID-19 the currently effective control methods include expanding the scale of nucleic acid detection and treatment. Hence, for our multigroup COVID-19 model we consider the control problem and use two strategies, that is, expanding the scale of nucleic acid detection and treatment.

For the proposed multigroup COVID-19 model we study the stability of disease-free equilibrium, and obtain that the disease-free equilibrium is globally asymptotically stable when the basic reproduction number is less than or equal to one, and the infection graph is strongly connected. When the diseasefree equilibrium is unstable, two control strategies are provided to make the disease-free equilibrium globally asymptotically stable, namely linear state feedback and finite-time controllers. Finally, we use the multigroup COVID-19 model to simulate the COVID-19 outbreak in Hubei, Guangdong and Zhejiang provinces in China.

The paper is organized as follows: Sect. 2 presents some mathematical notations, some facts about digraph, the problem statement, and two lemmas. Section 3.1 considers the stability of disease-free equilibrium. Section 3.2 studies the stability of endemic equilibrium. Section 3.3 presents some control strategies for the proposed multigroup COVID-19 model. Section 4 provides the simulation of the COVID-19 outbreak in Hubei, Guangdong and Zhejiang provinces in China. Section 5 collects our conclusions and ideas for future work.

\section{Preliminaries}

\subsection{Notations}

Let $\mathbb{R}^{n}$ denote the set of real $n$-dimensional column vectors. For $x=\left(x_{1}, \ldots, x_{n}\right)^{\top}$, let $\mathbb{R}_{+}^{n}:=\left\{x \mid x_{i} \geq\right.$ $0, \quad i=1, \ldots, n\}$. For a real square matrix $A, \rho(A)$ 
stands for the spectral radius of matrix $A$, which is the smallest real positive number such that $\rho(A) \geq$ $\left|\lambda_{i}(A)\right|, \quad \forall i=1, \ldots, n$, where $\lambda_{i}(A), i=1, \ldots, n$ are the eigenvalues of $A$. A matrix $A$ is irreducible if and only if it cannot be transformed into a block uppertriangular form by simultaneous row/column permutations.

\subsection{Some facts about digraph}

Let $\mathscr{G}=(\mathscr{V}, \mathscr{E}, A)$ be a weighted digraph (directed graph), where $\mathscr{V}=\{1,2, \ldots, N\}$ is the set of nodes, $\mathscr{E} \in \mathscr{V} \times \mathscr{V}$ is a set of edges, and $A=$ $\left(a_{i j}\right)_{N \times N}$ is an adjacency matrix which assigns real numbers to the edges. Let $\mathscr{G}(A)$ denote a graph corresponding to adjacency matrix $A$. A directed path (length $l-1$ ) is a sequence of directed edges of the form $\left(i_{1}, i_{2}\right),\left(i_{2}, i_{3}\right), \ldots,\left(i_{l 1}, i_{l}\right)$ with distinct nodes. A digraph is said to be strongly connected if there exists a directed path between any two distinct nodes. For an undirected graph, the adjacency matrix is symmetric. An undirected graph is said to be connected if there exists a path between any two distinct vertices of the graph. The adjacency matrix is irreducible if and only if the digraph (undirected graph) is strongly connected (connected).

\subsection{Problem statements}

In order to formulate a multigroup COVID-19 model with immunity, we partition the total population into $N$ groups, and divide the total population $W_{i}$ of $i$ th group into five compartments, that is, a susceptible compartment $S_{i}$, an exposed compartment $E_{i}$, an infective compartment $I_{i}$, an infective compartment in treatment $T_{i}$ and a recovered compartment $R_{i}$. Figure 1 depicts the flow diagram of multigroup COVID-19 model.

The model is presented as the following set of ODEs:

$$
\left\{\begin{array}{l}
\dot{S}_{i}=\left(1-p_{i}\right) b_{i}-\sum_{j=1}^{N} \frac{\alpha_{i j} S_{i} I_{j}}{1+\sigma I_{j}^{2}}-\mu_{i}^{S} S_{i}+\rho_{i} R_{i} \\
\dot{E}_{i}=\sum_{j=1}^{N} \frac{\alpha_{i j} S_{i} I_{j}}{1+\sigma I_{j}^{2}}-\left(\beta_{i}+\mu_{i}^{E}\right) E_{i} \\
\dot{I}_{i}=\beta_{i} E_{i}-\left(\gamma_{i}+\delta_{1 i}+\mu_{i}^{I}\right) I_{i} \\
\dot{T}_{i}=\gamma_{i} I_{i}-\left(\sigma_{i}+\delta_{2 i}+\mu_{i}^{T}\right) T_{i} \\
\dot{R}_{i}=p_{i} b_{i}+\sigma_{i} T_{i}-\left(\mu_{i}^{R}+\rho_{i}\right) R_{i}
\end{array}\right.
$$

where $i=1, \ldots, N$. All variables and parameters are described in Table 1. Let $\mathscr{G}(A)$ denote the infection graph of multigroup COVID-19 model, where

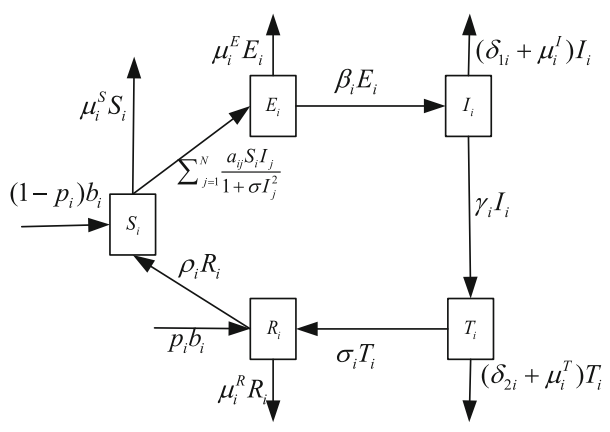

Fig. 1 Flow diagram of the multigroup COVID-19 model

$A=\left(\alpha_{i j}\right)_{N \times N}$. In model (1), we have the following important assumptions:

(1) The population in susceptible compartment enter into the exposed compartment by adequate contact with infective individuals not in treatment.

(2) Infective individuals not in treatment are infectious to the susceptible population.

(3) For different groups cross infection occurs between susceptible compartment and infective compartment.

(4) Part of the recovered compartment become susceptible again to the disease.

The assumptions (1)-(4) are reasonable for many epidemic diseases, such as measles, severe acute respiratory syndrome (SARS), mumps, gonorrhea [13]. Because the spread of COVID-19 and SARS is very similar, all assumptions (1)-(4) are reasonable for COVID-19. For a single group, the model (1) is investigated in [13], and the incidence rate is in bilinear form $\alpha S I$. However, this bilinear incidence rate may not be suitable for some epidemic diseases, such as SARS and COVID-19. As pointed out in [24], when SARS broke out early, the incidence rate is increasing when the infective population is small. When the number of infected people grows quickly, since the government has implemented aggressive measures and policies, such as border inspections, nucleic acid testing, mask wearing, isolation, etc., the incidence rate is decreasing as the infective population is large. In order to describe this phenomenon, the authors provided the following incidence rate

$\frac{\alpha I S}{1+\sigma I^{2}}$ 
Table 1 Variables and parameters of multigroup COVID-19 model

\begin{tabular}{|c|c|}
\hline Variable & Explanation \\
\hline$S_{i}$ & Susceptible population of $i$ th group \\
\hline$E_{i}$ & Exposed population of $i$ th group \\
\hline$I_{i}$ & Infective population of $i$ th group \\
\hline$T_{i}$ & Infective population in treatment of $i$ th group \\
\hline$R_{i}$ & Recovered population of $i$ th group \\
\hline$W_{i}$ & The total population in $i$ th group \\
\hline$b_{i}$ & $\begin{array}{l}\text { The rate at which new individual enter the population } \\
\text { of } i \text { th group }\end{array}$ \\
\hline$p_{i}$ & A fraction of $b_{i}$ has antibodies $\left(0 \leq p_{i}<1\right)$ \\
\hline$\alpha_{i j}$ & $\begin{array}{l}\text { The cross infection coefficient from } i \text { th group to } j \text { th } \\
\text { group }\end{array}$ \\
\hline$\sigma$ & $\begin{array}{l}\text { The parameter describing the psychological effect of } \\
\text { the general public toward the infectives }\end{array}$ \\
\hline$\mu_{i}^{S}$ & $\begin{array}{l}\text { The death rate of populations occurring in } S_{i} \text { com- } \\
\text { partment }\end{array}$ \\
\hline$\mu_{i}^{E}$ & $\begin{array}{l}\text { The death rate of populations occurring in } E_{i} \text { com- } \\
\text { partment }\end{array}$ \\
\hline$\mu_{i}^{I}$ & $\begin{array}{l}\text { The death rate of populations occurring in } I_{i} \text { compart- } \\
\text { ment }\end{array}$ \\
\hline$\mu_{i}^{T}$ & $\begin{array}{l}\text { The death rate of populations occurring in } T_{i} \text { com- } \\
\text { partment }\end{array}$ \\
\hline$\mu_{i}^{R}$ & $\begin{array}{l}\text { The death rate of populations occurring in } R_{i} \text { com- } \\
\text { partment }\end{array}$ \\
\hline$\delta_{1 i}$ & $\begin{array}{l}\text { The disease-related death rate of infective population } \\
\text { in } i \text { th group who are not in treatment }\end{array}$ \\
\hline$\delta_{2 i}$ & $\begin{array}{l}\text { The disease-related death rate of infective population } \\
\text { in } i \text { th group who are in treatment }\end{array}$ \\
\hline$\beta_{i}$ & $\begin{array}{l}\text { The transmission coefficient from exposed population } \\
\text { to infective population }\end{array}$ \\
\hline$\gamma_{i}$ & $\begin{array}{l}\text { The transmission coefficient from infective popula- } \\
\text { tion to treatment }\end{array}$ \\
\hline$\sigma_{i}$ & The recovery rate of infective population \\
\hline$\rho_{i}$ & $\begin{array}{l}\text { The proportion of recovered population who become } \\
\text { susceptible again to the disease }\end{array}$ \\
\hline
\end{tabular}

where $\alpha I$ describes the infection force of the disease and $\frac{1}{1+\sigma I^{2}}$ describes the effects of aggressive measures and policies.

Similar to the 2003 SARS outbreak, when COVID19 broke out, China enforced strict lockdown measures, such as losing all non-essential businesses and encouraging or mandating that people stay in their home. As the COVID-19 spreads in other countries, several countries have also implemented strict lockdown measures. These policies will lead to a decrease in the incidence rate due to the increase in the number of infections. Hence, the bilinear incidence rate is inappropriate for COVID-19. In order to accurately describe the characteristics of the incidence rate of COVID-19, we use the following incidence rate among different groups:

$\frac{\alpha_{i j} S_{i} I_{j}}{1+\sigma I_{j}^{2}}$.

Note that although the parameter $\sigma$ can describe the effects of aggressive measures and policies, the basic reproduction number has nothing to do with parameter $\sigma$ (see Sect. 3.1 for details). Hence, in order to completely control COVID-19, we need to design appropriate control strategies (see Sect. 4 for details). Although COVID-19 has become a pandemic worldwide, it has been died out in some areas, such as Hubei, Guangdong and Zhejiang provinces in China. Thus, COVID-19 can be completely controlled in some local areas.

Generally speaking, the model (1) exists a diseasefree equilibrium and an endemic equilibrium. When basic reproduction number is less than or equal to one, we will show that the disease-free equilibrium is globally asymptotically stable and the disease dies out. When the basic reproduction number is larger than one, the disease may persist in the population. Hence, when the basic reproduction number is larger than one, this paper aims to design appropriate feedback control such that the disease-free equilibrium is globally asymptotically stable.

\subsection{Some lemmas}

The following lemmas will be used in the Proof of Theorem 4.

Lemma 1 [25] Consider the system $\dot{x}=f(x)$, where $f(0)=0, x \in \mathbb{R}^{n}$. If there exist a positive definite function $V(x)\left(V(0)=0\right.$ and $\left.V(x)>0, \forall x \in \mathbb{R}^{n}, x \neq 0\right)$, numbers $c>0$ and $0<a<1$ such that $\dot{V}(x) \leq$ $-c(V(x))^{a}$. Then, $V(x)$ approaches 0 in finite time, and the setting time is estimated by $\frac{V(x(0))^{1-a}}{c(1-a)}$.

Lemma 2 [26] If $c_{1}, c_{2}, \ldots, c_{n}$ are positive numbers and $0<a \leq 1$, then

$\left(\sum_{i=1}^{n} c_{i}\right)^{a} \leq \sum_{i=1}^{n} c_{i}^{a}$. 


\section{Main results}

This section will study the property of model (1) and design appropriate control when the disease-free equilibrium is unstable. First, we will investigate the stability of the disease-free equilibrium. Second, we will study the stability of the endemic equilibrium. Third, when the disease-free equilibrium is unstable, we will design feedback control such that the disease-free equilibrium of closed-loop system is globally asymptotically stable.

\subsection{Stability analysis of disease-free equilibrium}

For a new model, we first need to study its well-posed problem. The following Proposition shows that the model (1) is well-posed epidemiologically and mathematically.

Proposition 1 Define feasible region $\Gamma$ as

$$
\begin{gathered}
\Gamma=\left\{\left(S_{1}, E_{1}, I_{1}, T_{1}, R_{1}, \ldots, S_{N}, E_{N}, I_{N}, T_{N}, R_{N}\right)^{\top}\right. \\
\left.\in \mathbb{R}_{+}^{5 N}: W_{i} \leq \frac{b_{i}}{\psi_{i}}, \quad i=1, \ldots, N\right\}
\end{gathered}
$$

where $W_{i}=S_{i}+E_{i}+I_{i}+T_{i}+R_{i}$ denotes the total number of high-risk human population in ith group, $\psi_{i}=\min \left\{\mu_{i}^{S}, \mu_{i}^{E}, \mu_{i}^{R}, \delta_{1 i}+\mu_{i}^{I}, \delta_{2 i}+\mu_{i}^{T}\right\}$. The feasible region $\Gamma$ is positively invariant with respect to the model (1).

Proof By adding the equations of the model (1), one can obtain

$$
\begin{aligned}
\dot{W}_{i}= & b_{i}-\mu_{i}^{S} S_{i}-\mu_{i}^{E} E_{i}-\left(\delta_{1 i}+\mu_{i}^{I}\right) I_{i} \\
& -\left(\delta_{2 i}+\mu_{i}^{T}\right) T_{i}-\mu_{i}^{R} R_{i} \\
& \leq b_{i}-\psi_{i} W_{i}
\end{aligned}
$$

One can obtain from (2) that

$$
W_{i} \leq e^{-\psi_{i} t} W_{i}(0)+\frac{b_{i}}{\psi_{i}}\left(1-e^{-\psi_{i} t}\right) .
$$

When $t \rightarrow \infty, W_{i} \leq \frac{b_{i}}{\psi_{i}}$.

In the sequel, we will show that the feasible region $\Gamma$ is positively invariant with respect to the model (1). One can obtain from system (1) that
$\dot{S}_{i} \geq-\left(\sum_{j=1}^{N} \frac{\alpha_{i j} I_{j}}{1+\sigma I_{j}^{2}}+\mu_{i}^{S}\right) S_{i}$.

Suppose that $m_{i}(t)=-\sum_{j=1}^{N} \frac{\alpha_{i j} I_{j}}{1+\sigma I_{j}^{2}}-\mu_{i}^{S}$ and $v_{i}(t)=$ $\exp \left(\int_{0}^{t} m_{i}(s) \mathrm{d} s\right)$. Then, one has $v_{i}(0)=1$ and $\dot{v}_{i}(t)=$ $m_{i}(t) v_{i}(t)$. It is easy to get

$\frac{\mathrm{d}}{\mathrm{d} t}\left(\frac{S_{i}}{v_{i}}\right)=\frac{\dot{S}_{i} v_{i}-S_{i} \dot{v}_{i}}{v_{i}^{2}}$, $\geq 0$.

Hence,

$S_{i} \geq S_{i}(0) \exp \left(\int_{0}^{t} m_{i}(s) \mathrm{d} s\right)$.

If $S_{i}(0) \geq 0$, then $S_{i}(t) \geq 0, \forall t \geq 0$.

One can obtain from the second equation of system (1) that

$\dot{E}_{i} \geq-\left(\beta_{i}+\mu_{i}^{E}\right) E_{i}$.

Then, it is easy to obtain

$E_{i}(t) \geq E_{i}(0) \exp \left(-\left(\beta_{i}+\mu_{i}^{E}\right) t\right)$.

If $E_{i}(0) \geq 0$, then $E_{i}(t) \geq 0, \forall t \geq 0$. Using the similar method, one can obtain that $I_{i}, T_{i}, R_{i}$ are nonnegative when $I_{i}(0), T_{i}(0), R_{i}(0)$ are nonnegative. Hence, the feasible region $\Gamma$ is positively invariant with respect to the model (1). This completes the Proof of Proposition 1.

It is easy to see that $P_{0}=\left(S_{1}^{0}, 0,0,0, R_{1}^{0}, \ldots, S_{N}^{0}\right.$, $\left.0,0,0, R_{N}^{0}\right)^{\top}$ is a disease-free equilibrium of the model (1), where $S_{i}^{0}=\frac{b_{i}\left[\left(1-p_{i}\right) \mu_{i}^{R}+\rho_{i}\right]}{\mu_{i}^{S}\left(\mu_{i}^{R}+\rho_{i}\right)}, R_{i}^{0}=\frac{p_{i} b_{i}}{\mu_{i}^{R}+\rho_{i}}$. For the multigroup COVID-19 model (1), we take the infected compartments to be $E_{i}$ and $I_{i}$. Let $\mathscr{F}_{i}$ and $\mathscr{Y}_{i}$ denote the rate of secondary infections increase and the rate of disease progression, death, recovery decrease in the $i$ th compartment respectively. Then

$$
\begin{aligned}
\mathscr{F}_{i} & =\left(\begin{array}{c}
\sum_{j=1}^{N} \frac{\alpha_{i j} S_{i} I_{j}}{1+\sigma I_{j}^{2}} \\
0
\end{array}\right), \\
\mathscr{Y}_{i} & =\left(\begin{array}{c}
\left(\beta_{i}+\mu_{i}^{E}\right) E_{i} \\
-\beta_{i} E_{i}+\left(\gamma_{i}+\delta_{1 i}+\mu_{i}^{I}\right) I_{i}
\end{array}\right) .
\end{aligned}
$$


As pointed out in [27], the next generation matrix of model (1) can be presented as $F Y^{-1}$, where $F=$ $\left(\frac{\partial \mathscr{F}_{i}}{\partial x_{j}}\left(P_{0}\right)\right), Y=\left(\frac{\partial \mathscr{Y}_{i}}{\partial x_{j}}\left(P_{0}\right)\right), x_{j}=\left(E_{j}, I_{j}\right)$. Then, the basic reproduction number of model (1) can be chosen as $\rho\left(F Y^{-1}\right)$. By direct calculation, $F=\left(f_{i j}\right)_{N \times N}$ and $Y=\operatorname{diag}\left\{y_{11}, \ldots, y_{N N}\right\}$ (a block diagonal matrix), where

$$
\begin{aligned}
f_{i j} & =\left(\begin{array}{cc}
0 & \alpha_{i j} S_{i}^{0} \\
0 & 0
\end{array}\right), \\
y_{i i} & =\left(\begin{array}{cc}
\beta_{i}+\mu_{i}^{E} & 0 \\
-\beta_{i} & \gamma_{i}+\delta_{1 i}+\mu_{i}^{I}
\end{array}\right) .
\end{aligned}
$$

It is easy to obtain that $Y^{-1}=\operatorname{diag}\left\{y_{11}^{-1}, \ldots, y_{N N}^{-1}\right\}$, where

$$
y_{i i}^{-1}=\left(\begin{array}{cc}
\frac{1}{\beta_{i}+\mu_{i}^{E}} & 0 \\
\frac{\beta_{i}}{\left(\beta_{i}+\mu_{i}^{E}\right)\left(\gamma_{i}+\delta_{1 i}+\mu_{i}^{I}\right)} & \frac{1}{\gamma_{i}+\delta_{1 i}+\mu_{i}^{I}}
\end{array}\right) .
$$

Hence, $F Y^{-1}=\left(f_{i j} y_{i i}^{-1}\right)_{N \times N}$, where

$$
f_{i j} y_{i i}^{-1}=\left(\begin{array}{cc}
\frac{\alpha_{i j} S_{i}^{0} \beta_{i}}{\left(\beta_{i}+\mu_{i}^{E}\right)\left(\gamma_{i}+\delta_{1 i}+\mu_{i}^{I}\right)} & \frac{\alpha_{i j} S_{i}^{0}}{\gamma_{i}+\delta_{1 i}+\mu_{i}^{I}} \\
0 & 0
\end{array}\right) .
$$

Let

$$
M_{0}=\left(\frac{\alpha_{i j} \beta_{i} b_{i}\left[\left(1-p_{i}\right) \mu_{i}^{S}+\rho_{i}\right]}{\mu_{i}^{S}\left(\mu_{i}^{R}+\rho_{i}\right)\left(\beta_{i}+\mu_{i}^{E}\right)\left(\gamma_{i}+\delta_{1 i}+\mu_{i}^{I}\right)}\right)_{N \times N} .
$$

Then, $M_{0}$ is the principal submatrix of $F Y^{-1}$, that is, there exists a permutation matrix $P$ such that

$$
P\left(F Y^{-1}\right) P^{\top}=\left(\begin{array}{cc}
M_{0} & B \\
C & D
\end{array}\right),
$$

where $B$ and $D$ are zero matrices with appropriate dimension. Hence, $\rho\left(M_{0}\right)=\rho\left(F Y^{-1}\right)$, and we define the basic reproduction number as $R_{0}=\rho\left(M_{0}\right)$.

The following theorem states that the disease-free equilibrium is globally stable under appropriate conditions.

Theorem 1 Suppose that the infection graph $\mathscr{G}(A)$ is strongly connected. If $R_{0} \leq 1$, then the diseasefree equilibrium $P_{0}$ is globally asymptotically stable. If $R_{0}>1$, then the disease-free equilibrium $P_{0}$ is unstable.
Proof When the infection graph $\mathscr{G}(A)$ is strongly connected, the adjacency matrix $A=\left(\alpha_{i j}\right)_{N \times N}$ is irreducible, and the nonnegative matrix $M_{0}$ is also irreducible. By Perron-Frobenius Theorem, the spectral radius $\rho\left(M_{0}\right)>0$ is an algebraically simple eigenvalue of $M_{0}$, and the corresponding left eigenvector $w=\left(w_{1}, w_{2}, \ldots, w_{N}\right)^{\top}$ is positive. Define

$V(t)=\sum_{i=1}^{N} \frac{w_{i}\left[\beta_{i} E_{i}+\left(\beta_{i}+\mu_{i}^{E}\right) I_{i}\right]}{\left(\beta_{i}+\mu_{i}^{E}\right)\left(\gamma_{i}+\delta_{1 i}+\mu_{i}^{I}\right)}$.

$V(t)$ is nonnegative for all $t \geq 0$, and $V(t)=0$ if and only if $E_{i}=I_{i}=0, i=1, \ldots, N$. Hence, $V(t)$ is a good candidate for Lyapunov function. The time derivative of (5) along system (1) is

$$
\begin{aligned}
\dot{V} & =\sum_{i=1}^{N} \frac{w_{i}\left[\beta_{i} \dot{E}_{i}+\left(\beta_{i}+\mu_{i}^{E}\right) \dot{I}_{i}\right]}{\left(\beta_{i}+\mu_{i}^{E}\right)\left(\gamma_{i}+\delta_{1 i}+\mu_{i}^{I}\right)}, \\
& =\sum_{i=1}^{N} w_{i}\left[\frac{\beta_{i}}{\left(\beta_{i}+\mu_{i}^{E}\right)\left(\gamma_{i}+\delta_{1 i}+\mu_{i}^{I}\right)} \sum_{j=1}^{N} \frac{\alpha_{i j} S_{i} I_{j}}{1+\sigma I_{j}^{2}}-I_{i}\right], \\
& \leq \sum_{i=1}^{N} w_{i}\left[\frac{\beta_{i}}{\left(\beta_{i}+\mu_{i}^{E}\right)\left(\gamma_{i}+\delta_{1 i}+\mu_{i}^{I}\right)} \sum_{j=1}^{N} \frac{\alpha_{i j} S_{i}^{0} I_{j}}{1+\sigma I_{j}^{2}}-I_{i}\right], \\
& \leq \sum_{i=1}^{N} w_{i}\left[\frac{\beta_{i} b_{i}\left[\left(1-p_{i}\right) \mu_{i}^{R}+\rho_{i}\right]}{\mu_{i}^{S}\left(\mu_{i}^{R}+\rho_{i}\right)\left(\beta_{i}+\mu_{i}^{E}\right)\left(\gamma_{i}+\delta_{1 i}+\mu_{i}^{I}\right)} \sum_{j=1}^{N} \alpha_{i j} I_{j}-I_{i}\right], \\
& =w^{\top}\left(M_{0} I-I\right), \\
& =\left(\rho\left(M_{0}\right)-1\right) w^{\top} I,
\end{aligned}
$$

where $I=\left(I_{1}, I_{2}, \ldots, I_{N}\right)^{\top}$. If $\rho\left(M_{0}\right) \leq 1$, then $\dot{V} \leq$ 0. If $\rho\left(M_{0}\right)<1$, then $\dot{V}=0$ if and only if $I=0$. If $\rho\left(M_{0}\right)=1$, then $\dot{V}=0$ implies that

$$
\begin{aligned}
& \sum_{i=1}^{N} w_{i}\left[\frac{\beta_{i}}{\left(\beta_{i}+\mu_{i}^{E}\right)\left(\gamma_{i}+\delta_{1 i}+\mu_{i}^{I}\right)} \sum_{j=1}^{N} \alpha_{i j} S_{i} I_{j}\right] \\
& \quad=\sum_{i=1}^{N} w_{i} I_{i} .
\end{aligned}
$$

Since

$$
\begin{aligned}
& \sum_{i=1}^{N} w_{i}\left[\frac{\beta_{i}}{\left(\beta_{i}+\mu_{i}^{E}\right)\left(\gamma_{i}+\delta_{1 i}+\mu_{i}^{I}\right)} \sum_{j=1}^{N} \alpha_{i j} S_{i} I_{j}\right] \\
& \quad \leq \sum_{i=1}^{N} w_{i}\left[\frac{\beta_{i}}{\left(\beta_{i}+\mu_{i}^{E}\right)\left(\gamma_{i}+\delta_{1 i}+\mu_{i}^{I}\right)} \sum_{j=1}^{N} \alpha_{i j} S_{i}^{0} I_{j}\right] \\
& =w^{\top} M_{0} I,
\end{aligned}
$$


the above equal sign holds if and only $S_{i}=S_{i}^{0}, i=$ $1, \ldots, N$. Hence, if $\rho\left(M_{0}\right)=1$, then $\dot{V}=0$ implies that $I=0$ or $S_{i}=S_{i}^{0}, i=1, \ldots, N$. It is easy to see that $\dot{V}=0$ if and only if $\left(S_{1}, E_{1}, I_{1}, T_{1}, R_{1}, \ldots, S_{N}\right.$, $\left.E_{N}, I_{N}, T_{N}, R_{N}\right)^{\top}=P_{0}$. Hence, by LaSalle's Invariance Principle, the disease-free equilibrium $P_{0}$ is globally asymptotically stable.

One can see from the above proof that $\dot{V}=$ $\left(\rho\left(M_{0}\right)-1\right) w^{\top} I=0$ at $P_{0}$. If $\rho\left(M_{0}\right)>1$ and in a neighborhood of $P_{0}$, then $\dot{V}>0$. Hence, the diseasefree equilibrium $P_{0}$ is unstable when $\rho\left(M_{0}\right)>1$. This completes the Proof of Theorem 1.

\subsection{Stability analysis of endemic equilibrium}

An equilibrium $P^{*}=\left(S_{1}^{*}, E_{1}^{*}, I_{1}^{*}, T_{1}^{*}, R_{1}^{*}, \ldots\right.$, $\left.T_{N}^{*}, R_{N}^{*}\right)^{\top}$ in the interior of $\Gamma$ is called an endemic equilibrium, where $P^{*}$ satisfies

$$
\left\{\begin{array}{l}
\left(1-p_{i}\right) b_{i}-\sum_{j=1}^{N} \frac{\alpha_{i j} S_{i}^{*} I_{j}^{*}}{1+\sigma I_{j}^{* 2}}-\mu_{i}^{S} S_{i}^{*}+\rho_{i} R_{i}^{*}=0 \\
\sum_{j=1}^{N} \frac{\alpha_{i j} S_{i}^{*} I_{j}^{*}}{1+\sigma I_{j}^{* 2}}-\left(\beta_{i}+\mu_{i}^{E}\right) E_{i}^{*}=0 \\
\beta_{i} E_{i}^{*}-\left(\gamma_{i}+\delta_{1 i}+\mu_{i}^{I}\right) I_{i}^{*}=0 \\
\gamma_{i} I_{i}^{*}-\left(\sigma_{i}+\delta_{2 i}+\mu_{i}^{T}\right) T_{i}^{*}=0 \\
p_{i} b_{i}+\sigma_{i} T_{i}^{*}-\left(\mu_{i}^{R}+\rho_{i}\right) R_{i}^{*}=0 \\
i=1,2, \ldots, N
\end{array}\right.
$$

If $N=1$ and $\sigma=0$, then system (1) has a unique endemic equilibrium when $\rho\left(M_{0}\right)>1[13$, see the Theorem 3]. However, when $N \geq 2$, the system (1) may have multiple endemic equilibriums. If the assumption (4) does not hold, that is, $\rho_{i}=0, i=1, \ldots, N$, then the endemic equilibrium $P^{*}$ is unique and globally asymptotically stable under appropriate conditions.

Theorem 2 Suppose that the infection graph $\mathscr{G}(A)$ is strongly connected, and $P^{*}$ is an arbitrary endemic equilibrium. If $\rho_{i}=0, i=1, \ldots, N, R_{0}>1$, and

$\sigma I_{j} I_{j}^{*}-1 \leq 0, \quad \forall I_{j}>0, \quad j=1, \ldots, N$

then there exists a unique endemic equilibrium $P^{*}$, and $P^{*}$ is globally asymptotically stable in the interior of $\Gamma$.

Proof When $\rho_{i}=0, i=1, \ldots, N$, the variables $T_{i}, R_{i}$ do not exist in the first three equations of (1). Hence, we just need to consider the equations about $S_{i}, E_{i}, I_{i}$. Let $g_{i j}\left(S_{i}, I_{j}\right)=\frac{S_{i} I_{j}}{1+\sigma I_{j}^{2}}$.

Then for all $S_{i} \neq S_{i}^{*}$,

$$
\begin{aligned}
& \left(S_{i}-S_{i}^{*}\right)\left[g_{i i}\left(S_{i}, I_{i}^{*}\right)-g_{i i}\left(S_{i}^{*}, I_{i}^{*}\right)\right] \\
& =\left(S_{i}-S_{i}^{*}\right)^{2} \frac{I_{i}^{*}}{1+\sigma\left(I_{i}^{*}\right)^{2}} \\
& >0 .
\end{aligned}
$$

If the condition (7) holds, then through direct calculation, we have

$$
\begin{aligned}
& {\left[g_{i i}\left(S_{i}^{*}, I_{i}^{*}\right) g_{i j}\left(S_{i}, I_{j}\right)-g_{i j}\left(S_{i}^{*}, I_{j}^{*}\right) g_{i i}\left(S_{i}, I_{i}^{*}\right)\right] .} \\
& {\left[\frac{g_{i i}\left(S_{i}^{*}, I_{i}^{*}\right) g_{i j}\left(S_{i}, I_{j}\right)}{I_{j}}-\frac{g_{i j}\left(S_{i}^{*}, I_{j}^{*}\right) g_{i i}\left(S_{i}, I_{i}^{*}\right)}{I_{j}^{*}}\right] \leq 0 .}
\end{aligned}
$$

Hence, the conditions of Theorem 2.2 in [9] hold. Let

$$
\begin{aligned}
V_{i}= & \int_{S_{i}^{*}}^{S_{i}} \frac{g_{i i}\left(\tau, I_{i}^{*}\right)-g_{i i}\left(S_{i}^{*}, I_{i}^{*}\right)}{g_{i i}\left(\tau, I_{i}^{*}\right)} d \tau \\
& +\left(E_{i}-E_{i}^{*} \ln E_{i}\right)+\left(I_{i}-I_{i}^{*} \ln I_{i}\right) .
\end{aligned}
$$

Similar to the Proof of Theorem 2.2 [9], we can obtain that there exists a unique endemic equilibrium $P^{*}$, and $P^{*}$ is globally asymptotically stable in the interior of $\Gamma$. This completes the Proof of Theorem 2.

If the assumption (4) holds, that is, $\rho_{i} \neq 0, i=$ $1, \ldots, N$, then it is hard to obtain that the endemic equilibrium $P^{*}$ is unique and globally asymptotically stable. There may exist multiple endemic equilibrium points, and the dynamic behavior of the system (1) will be very rich. For example, the system (1) may exist bifurcation phenomenon. In this case, it may be more difficult to control the spread of COVID-19. What is the impact of multiple endemic equilibrium points on the spread of COVID-19? We will try to solve it in future work.

\subsection{Multigroup COVID-19 model with control}

If $R_{0}>1$, then the disease-free equilibrium $P_{0}$ is unstable. In this case, we want to design appropriate control such that the disease-free equilibrium of closed-loop system is globally asymptotically stable, that is, the disease dies out under appropriate control. In reality, the 
government can reduce the number of exposed people and increase the number of people treated such that the disease dies out. Suppose that partially exposed people can be tested to eliminate the risk of infection, such as temperature and pharynx swab test for COVID-19. Suppose that the excluded rate (the number of newly excluded exposed population per unit time) is given as a function of $E_{i}$ by $u_{i}^{E}\left(E_{i}\right)$, and the treatment rate (the number of newly added treatment population per unit time) is given as a function of $I_{i}$ by $u_{i}^{I}\left(I_{i}\right)$. Then, the epidemic model (1) with two controllers becomes

$$
\left\{\begin{array}{l}
\dot{S}_{i}=\left(1-p_{i}\right) b_{i}-\sum_{j=1}^{N} \frac{\alpha_{i j} S_{i} I_{j}}{1+\sigma I_{j}^{2}}-\mu_{i}^{S} S_{i}+\rho_{i} R_{i} \\
\dot{E}_{i}=\sum_{j=1}^{N} \frac{\alpha_{i j} S_{i} I_{j}}{1+\sigma I_{j}^{2}}-\left(\beta_{i}+\mu_{i}^{E}\right) E_{i}-u_{i}^{E}\left(E_{i}\right) \\
\dot{I}_{i}=\beta_{i} E_{i}-\left(\gamma_{i}+\delta_{1 i}+\mu_{i}^{I}\right) I_{i}-u_{i}^{I}\left(I_{i}\right) \\
\dot{T}_{i}=\gamma_{i} I_{i}+u_{i}^{I}\left(I_{i}\right)-\left(\sigma_{i}+\delta_{2 i}+\mu_{i}^{T}\right) T_{i} \\
\dot{R}_{i}=p_{i} b_{i}+u_{i}^{E}\left(E_{i}\right)+\sigma_{i} T_{i}-\left(\mu_{i}^{R}+\rho_{i}\right) R_{i}
\end{array}\right.
$$

where $i=1, \ldots, N$.

The most natural choice is linear state feedback. The Algorithm 1 provides a systematic method to identify the groups that need to be controlled.

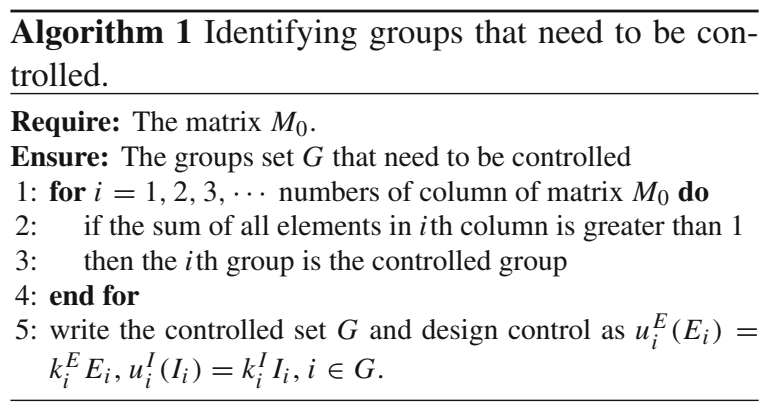

The following theorem states that the disease-free equilibrium of closed-loop system (8) is globally asymptotically stable. Let $M_{0}=\left(m_{i j}\right)_{N \times N}$, where

$m_{i j}=\frac{\alpha_{i j} \beta_{i} b_{i}\left[\left(1-p_{i}\right) \mu_{i}^{S}+\rho_{i}\right]}{\mu_{i}^{S}\left(\mu_{i}^{R}+\rho_{i}\right)\left(\beta_{i}+\mu_{i}^{E}\right)\left(\gamma_{i}+\delta_{1 i}+\mu_{i}^{I}\right)}$.

Theorem 3 Consider the epidemic model (8) with two controllers provided in Algorithm 1. Suppose that the infection graph $\mathscr{G}(A)$ is strongly connected, and $R_{0}>$ 1. If $k_{i}^{E}>0$ and

$$
k_{i}^{I}>\left(\sum_{j=1}^{N} m_{j i}-1\right)\left(\gamma_{i}+\delta_{1 i}+\mu_{i}^{I}\right), \quad i \in G,
$$

then the disease-free equilibrium of closed-loop system (8) is globally asymptotically stable.

Proof When the spectral radius $\rho\left(M_{0}\right)>1$ and there does not exist controllers, by the Theorem 1, the disease-free equilibrium $P_{0}$ is unstable. For the epidemic model (8) with two controllers, the disease-free equilibrium is also $P_{0}$. Define

$V(t)=\sum_{i=1}^{N} \frac{\beta_{i} E_{i}+\left(\beta_{i}+\mu_{i}^{E}\right) I_{i}}{\left(\beta_{i}+\mu_{i}^{E}\right)\left(\gamma_{i}+\delta_{1 i}+\mu_{i}^{I}\right)}$.

The time derivative of (10) along system (8) is

$$
\begin{aligned}
& \dot{V}=\sum_{i=1}^{N} \frac{\beta_{i} \dot{E}_{i}+\left(\beta_{i}+\mu_{i}^{E}\right) \dot{I}_{i}}{\left(\beta_{i}+\mu_{i}^{E}\right)\left(\gamma_{i}+\delta_{1 i}+\mu_{i}^{I}\right)}, \\
& =\sum_{i=1}^{N}\left[\frac{\beta_{i}}{\left(\beta_{i}+\mu_{i}^{E}\right)\left(\gamma_{i}+\delta_{1 i}+\mu_{i}^{I}\right)} \sum_{j=1}^{N} \frac{\alpha_{i j} S_{i} I_{j}}{1+\sigma I_{j}^{2}}-I_{i}\right] \\
& -\sum_{i=1}^{N} \frac{\left[\beta_{i} k_{i}^{E} E_{i}+\left(\beta_{i}+\mu_{i}^{E}\right) k_{i}^{I} I_{i}\right]}{\left(\beta_{i}+\mu_{i}^{E}\right)\left(\gamma_{i}+\delta_{1 i}+\mu_{i}^{I}\right)}, \\
& \leq \sum_{i=1}^{N}\left[\frac{\beta_{i}}{\left(\beta_{i}+\mu_{i}^{E}\right)\left(\gamma_{i}+\delta_{1 i}+\mu_{i}^{I}\right)} \sum_{j=1}^{N} \alpha_{i j} S_{i}^{0} I_{j}-I_{i}\right] \\
& -\sum_{i=1}^{N} \frac{\left[\beta_{i} k_{i}^{E} E_{i}+\left(\beta_{i}+\mu_{i}^{E}\right) k_{i}^{I} I_{i}\right]}{\left(\beta_{i}+\mu_{i}^{E}\right)\left(\gamma_{i}+\delta_{1 i}+\mu_{i}^{I}\right)}, \\
& =\sum_{i=1}^{N}\left(\sum_{j=1}^{N} m_{i j} I_{j}-I_{i}\right) \\
& -\sum_{i=1}^{N} \frac{\left[\beta_{i} k_{i}^{E} E_{i}+\left(\beta_{i}+\mu_{i}^{E}\right) k_{i}^{I} I_{i}\right]}{\left(\beta_{i}+\mu_{i}^{E}\right)\left(\gamma_{i}+\delta_{1 i}+\mu_{i}^{I}\right)}, \\
& =\sum_{i=1}^{N}\left(\sum_{j=1}^{N} m_{j i}-1\right) I_{i} \\
& -\sum_{i=1}^{N} \frac{\left[\beta_{i} k_{i}^{E} E_{i}+\left(\beta_{i}+\mu_{i}^{E}\right) k_{i}^{I} I_{i}\right]}{\left(\beta_{i}+\mu_{i}^{E}\right)\left(\gamma_{i}+\delta_{1 i}+\mu_{i}^{I}\right)} .
\end{aligned}
$$

Since the controllers are obtained by Algorithm $1, k_{i}^{E}=$ $k_{i}^{I}=0, \forall i \notin G$. By the Algorithm $1, \sum_{j=1}^{N} m_{j i}<$ $1, \forall i \notin G$. Then

$$
\sum_{i=1}^{N}\left(\sum_{j=1}^{N} m_{j i}-1\right) I_{i}
$$




$$
\begin{aligned}
& -\sum_{i=1}^{N} \frac{\left[\beta_{i} k_{i}^{E} E_{i}+\left(\beta_{i}+\mu_{i}^{E}\right) k_{i}^{I} I_{i}\right]}{\left(\beta_{i}+\mu_{i}^{E}\right)\left(\gamma_{i}+\delta_{1 i}+\mu_{i}^{I}\right)} \\
= & \sum_{i \in G}\left[\sum_{j=1}^{N} m_{j i}-1-\frac{k_{i}^{I}}{\gamma_{i}+\delta_{1 i}+\mu_{i}^{I}}\right] I_{i} \\
& +\sum_{i \notin G}\left(\sum_{j=1}^{N} m_{j i}-1\right) I_{i} \\
& -\sum_{i \in G} \frac{\beta_{i} k_{i}^{E} E_{i}}{\left(\beta_{i}+\mu_{i}^{E}\right)\left(\gamma_{i}+\delta_{1 i}+\mu_{i}^{I}\right)} .
\end{aligned}
$$

When the condition (9) holds, then $\dot{V} \leq 0$ and $\dot{V}=0$ if and only if $I_{i}=0, i=1, \ldots, N$. Hence, by LaSalle's Invariance Principle, the disease-free equilibrium $P_{0}$ of closed-loop system (8) is globally asymptotically stable. This completes the Proof of Theorem 3.

Although the linear state feedback controllers is a natural choice, there may take a long time to approach the disease-free equilibrium. If we design controllers as $\bar{u}_{i}^{E}\left(E_{i}\right)=\bar{k}_{i}^{E}\left(E_{i}\right)^{a}, \bar{u}_{i}^{I}\left(I_{i}\right)=\bar{k}_{i}^{I}\left(I_{i}\right)^{a}$, where $i \in G$ and $0<a<1$ is a constant, then the time required for convergence is finite. We call these controllers as finite-time controllers. The following theorem states that under appropriate choice of $\bar{k}_{i}^{E}, \bar{k}_{i}^{I}$ the diseasefree equilibrium of closed-loop system (8) is globally asymptotically stable, and the convergence can be achieved in finite time.

Theorem 4 Consider the epidemic model (8) with two controllers $\bar{u}_{i}^{E}\left(E_{i}\right)=\bar{k}_{i}^{E}\left(E_{i}\right)^{a}, \bar{u}_{i}^{I}\left(I_{i}\right)=\bar{k}_{i}^{I}\left(I_{i}\right)^{a}$, where $i \in G$ and $0<a<1$ is a constant. Suppose that the infection graph $\mathscr{G}(A)$ is strongly connected, and $R_{0}>1$. If $\bar{k}_{i}^{E}>0$ and

$\bar{k}_{i}^{I}>\left(\sum_{j=1}^{N} m_{j i}-1\right)\left(S_{i}^{0}\right)^{1-a}\left(\gamma_{i}+\delta_{1 i}+\mu_{i}^{I}\right), \quad i \in G$,

then the disease-free equilibrium of closed-loop system (8) is globally asymptotically stable, and the exposed compartment $E_{i}, i \in G$ and infective compartment $I_{i}, i \in G$ can achieve zero in finite time.

Proof If the spectral radius $\rho\left(M_{0}\right)>1$ and there does not exist controllers, one can know from the Theorem 1 that the disease-free equilibrium $P_{0}$ is unstable. For the epidemic model (8) with two controllers
$\bar{u}_{i}^{E}\left(E_{i}\right)=\bar{k}_{i}^{E}\left(E_{i}\right)^{a}, \bar{u}_{i}^{I}\left(I_{i}\right)=\bar{k}_{i}^{I}\left(I_{i}\right)^{a}$, the disease-free equilibrium is also $P_{0}$. Define

$V(t)=\sum_{i \in G} \frac{\beta_{i} E_{i}+\left(\beta_{i}+\mu_{i}^{E}\right) I_{i}}{\left(\beta_{i}+\mu_{i}^{E}\right)\left(\gamma_{i}+\delta_{1 i}+\mu_{i}^{I}\right)}$.

The time derivative of (12) along system (8) is

$$
\begin{aligned}
\dot{V}= & \sum_{i \in G} \frac{\beta_{i} \dot{E}_{i}+\left(\beta_{i}+\mu_{i}^{E}\right) \dot{I}_{i}}{\left(\beta_{i}+\mu_{i}^{E}\right)\left(\gamma_{i}+\delta_{1 i}+\mu_{i}^{I}\right)}, \\
= & \sum_{i \in G}\left[\frac{\beta_{i}}{\left(\beta_{i}+\mu_{i}^{E}\right)\left(\gamma_{i}+\delta_{1 i}+\mu_{i}^{I}\right)} \sum_{j=1}^{N} \frac{\alpha_{i j} S_{i} I_{j}}{1+\sigma I_{j}^{2}}-I_{i}\right] \\
& -\sum_{i \in G} \frac{\left[\beta_{i} \bar{k}_{i}^{E}\left(E_{i}\right)^{a}+\left(\beta_{i}+\mu_{i}^{E}\right) \bar{k}_{i}^{I}\left(I_{i}\right)^{a}\right]}{\left(\beta_{i}+\mu_{i}^{E}\right)\left(\gamma_{i}+\delta_{1 i}+\mu_{i}^{I}\right)}, \\
\leq & \sum_{i \in G}\left[\frac{\beta_{i}}{\left(\beta_{i}+\mu_{i}^{E}\right)\left(\gamma_{i}+\delta_{1 i}+\mu_{i}^{I}\right)} \sum_{j=1}^{N} \alpha_{i j} S_{i}^{0} I_{j}-I_{i}\right] \\
& -\sum_{i \in G} \frac{\left[\beta_{i} \bar{k}_{i}^{E}\left(E_{i}\right)^{a}+\left(\beta_{i}+\mu_{i}^{E}\right) \bar{k}_{i}^{I}\left(I_{i}\right)^{a}\right]}{\left(\beta_{i}+\mu_{i}^{E}\right)\left(\gamma_{i}+\delta_{1 i}+\mu_{i}^{I}\right)}, \\
= & \sum_{i \in G}\left(\sum_{j=1}^{N} m_{j i}-1\right) I_{i} \\
& -\sum_{i \in G} \frac{\left[\beta_{i} \bar{k}_{i}^{E}\left(E_{i}\right)^{a}+\left(\beta_{i}+\mu_{i}^{E}\right) \bar{k}_{i}^{I}\left(I_{i}\right)^{a}\right]}{\left(\beta_{i}+\mu_{i}^{E}\right)\left(\gamma_{i}+\delta_{1 i}+\mu_{i}^{I}\right)}, \\
= & \sum_{i \in G}\left(\sum_{j=1}^{N} m_{j i}-1\right) I_{i} \\
& -\sum_{i \in G} \frac{\bar{k}_{i}^{I}\left(I_{i}\right)^{a}}{\gamma_{i}+\delta_{1 i}+\mu_{i}^{I}-\sum_{i \in G} \frac{\beta_{i} \bar{k}_{i}^{E}\left(E_{i}\right)^{a}}{\left(\beta_{i}+\mu_{i}^{E}\right)\left(\gamma_{i}+\delta_{1 i}+\mu_{i}^{I}\right)} .} .
\end{aligned}
$$

By the Algorithm $1, \sum_{j=1}^{N} m_{j i}>1, \forall i \in G$. When the condition (11) holds,

$$
\begin{aligned}
& \sum_{i \in G}\left(\sum_{j=1}^{N} m_{j i}-1\right) I_{i}-\sum_{i \in G} \frac{\bar{k}_{i}^{I}\left(I_{i}\right)^{a}}{\gamma_{i}+\delta_{1 i}+\mu_{i}^{I}} \\
& \quad \leq \sum_{i \in G}\left[\left(\sum_{j=1}^{N} m_{j i}-1\right)\left(S_{i}^{0}\right)^{1-a}-\frac{\bar{k}_{i}^{I}}{\gamma_{i}+\delta_{1 i}+\mu_{i}^{I}}\right]\left(I_{i}\right)^{a} \\
& \quad \leq 0 .
\end{aligned}
$$

There exists $c>0$ such that

$$
\begin{gathered}
\sum_{i \in G}\left[\left(\sum_{j=1}^{N} m_{j i}-1\right)\left(S_{i}^{0}\right)^{1-a}\right. \\
\left.-\frac{\bar{k}_{i}^{I}}{\gamma_{i}+\delta_{1 i}+\mu_{i}^{I}}\right]\left(I_{i}\right)^{a}
\end{gathered}
$$




$$
\begin{aligned}
& -\sum_{i \in G} \frac{\beta_{i} \bar{k}_{i}^{E}\left(E_{i}\right)^{a}}{\left(\beta_{i}+\mu_{i}^{E}\right)\left(\gamma_{i}+\delta_{1 i}+\mu_{i}^{I}\right)} \\
& \leq-c\left[\sum_{i \in G} \frac{\left(I_{i}\right)^{a}}{\left(\gamma_{i}+\delta_{1 i}+\mu_{i}^{I}\right)^{a}}\right. \\
& \left.+\sum_{i \in G} \frac{\beta_{i}^{a}\left(E_{i}\right)^{a}}{\left(\beta_{i}+\mu_{i}^{E}\right)^{a}\left(\gamma_{i}+\delta_{1 i}+\mu_{i}^{I}\right)^{a}}\right], \\
& \leq-c V^{a},
\end{aligned}
$$

where the second inequality is obtain by Lemma 2 . Hence,

$\dot{V} \leq-c V^{a}$.

By Lemma 1, one can obtain that $V(t)$ approaches 0 in finite time, and the setting time is estimated by $\frac{V(0)^{1-a}}{c(1-a)}$. Hence, the exposed compartment $E_{i}, i \in G$ and infective compartment $I_{i}, i \in G$ can achieve zero in finite time, and the setting time is estimated by $\frac{V(0)^{1-a}}{c(1-a)}$.

In addition, similar to the Proof of Theorem 3, one can obtain that the disease-free equilibrium $P_{0}$ of closed-loop system (8) is globally asymptotically stable, and the details are omitted here for brevity. This completes the Proof of Theorem 4.

Remark 1 Note that when the epidemic model (8) has two controllers $\bar{u}_{i}^{E}\left(E_{i}\right)=\bar{k}_{i}^{E}\left(E_{i}\right)^{a}, \bar{u}_{i}^{I}\left(I_{i}\right)=\bar{k}_{i}^{I}\left(I_{i}\right)^{a}$, where $i \in G$ and $0<a<1$ is a constant, the closedloop system does not satisfy Existence and Uniqueness Theorem. However, since the two controllers are continuous, one can know from Peano's Existence Theorem and Extension Theorem that the system (8) exists at least one solution on $[0, \infty)$ for any initial state in $\Gamma$.

Remark 2 Note that in the Proof of Theorem 4, the constant $c$ can be chosen as $c=\min \left\{c_{1}, c_{2}\right\}$, where

$$
\begin{aligned}
c_{1}= & \min _{i \in G}\left(\gamma_{i}+\delta_{1 i}+\mu_{i}^{I}\right)^{a} \min _{i \in G}\left[\left(1-\sum_{j=1}^{N} m_{j i}\right)\left(S_{i}^{0}\right)^{1-a}\right. \\
& \left.+\frac{\bar{k}_{i}^{I}}{\gamma_{i}+\delta_{1 i}+\mu_{i}^{I}}\right], \\
c_{2}= & \min _{i \in G} \frac{\beta_{i}^{a}}{\left(\beta_{i}+\mu_{i}^{E}\right)^{a}\left(\gamma_{i}+\delta_{1 i}+\mu_{i}^{I}\right)^{a}} \\
& \times \min _{i \in G} \frac{\beta_{i} \bar{k}_{i}^{E}}{\left(\beta_{i}+\mu_{i}^{E}\right)\left(\gamma_{i}+\delta_{1 i}+\mu_{i}^{I}\right)} .
\end{aligned}
$$

The setting time is estimated by

$$
\frac{1}{c(1-a)}\left[\frac{\beta_{i} E_{i}(0)+\left(\beta_{i}+\mu_{i}^{E}\right) I_{i}(0)}{\left(\beta_{i}+\mu_{i}^{E}\right)\left(\gamma_{i}+\delta_{1 i}+\mu_{i}^{I}\right)}\right]^{1-a} .
$$

Remark 3 In reality, one needs to consider the required energy cost of different controllers. Let $f\left(x_{1}\right)=$ $\int_{0}^{x_{1}} k x d x, g\left(x_{1}\right)=\int_{0}^{x_{1}} k x^{a} d x$, where $k>0, x_{1}>0$ and $0<a<1$ are constants. Then, it is easy to obtain that $f\left(x_{1}\right)>g\left(x_{1}\right), x_{1}>1$ and $f\left(x_{1}\right)<g\left(x_{1}\right), x_{1}<$ 1 . The functions $f, g$ can be seen as cost of energy. Hence, when the gain are the same and $I_{i}>1, E_{i}>1$ the linear state feedback controllers need much energy compared to the finite-time controllers. If the gain are the same and $I_{i}<1, E_{i}<1$ the linear state feedback controllers need less energy compared to the finite-time controllers. If we define two new switching controllers

$$
\begin{gathered}
u_{i}^{S E}\left(E_{i}\right)= \begin{cases}\bar{k}_{i}^{E}\left(E_{i}\right)^{a}, & E_{i} \geq 1, \\
k_{i}^{E}\left(E_{i}\right), & E_{i}<1,\end{cases} \\
u_{i}^{S I}\left(I_{i}\right)= \begin{cases}\bar{k}_{i}^{I}\left(I_{i}\right)^{a}, & I_{i} \geq 1, \\
k_{i}^{I}\left(I_{i}\right), & I_{i}<1,\end{cases}
\end{gathered}
$$

then the switching controllers (13) can minimize the amount of the required energy cost. One can see reference [28] for more details about the energy of control of complex networks.

\section{Simulating the COVID-19 outbreak in Hubei, Guangdong and Zhejiang provinces}

This section will provide a simulation of the COVID-19 outbreak in Hubei, Guangdong and Zhejiang provinces in China to illustrate the effectiveness of the obtained results. Consider a multigroup COVID-19 model (1) with three groups which represent Hubei, Guangdong and Zhejiang provinces, respectively. In order to apply the model (1) to stimulate the progression of COVID19 in Hubei, Guangdong and Zhejiang provinces, we need to estimate the parameters in Table 1. The topology of cross infection among Hubei, Guangdong and Zhejiang provinces is provided in Fig. 2. Because there is no direct data about cross infection coefficient among three provinces, we set the cross infection coefficients as the migration ratio of the Spring Festival in 2020 which are presented in Fig. 2, and these data come from the Baidu migration map (https://qianxi.baidu. com/2020/). 
Fig. 2 The topology of cross infection among Hubei, Guangdong and Zhejiang provinces in China

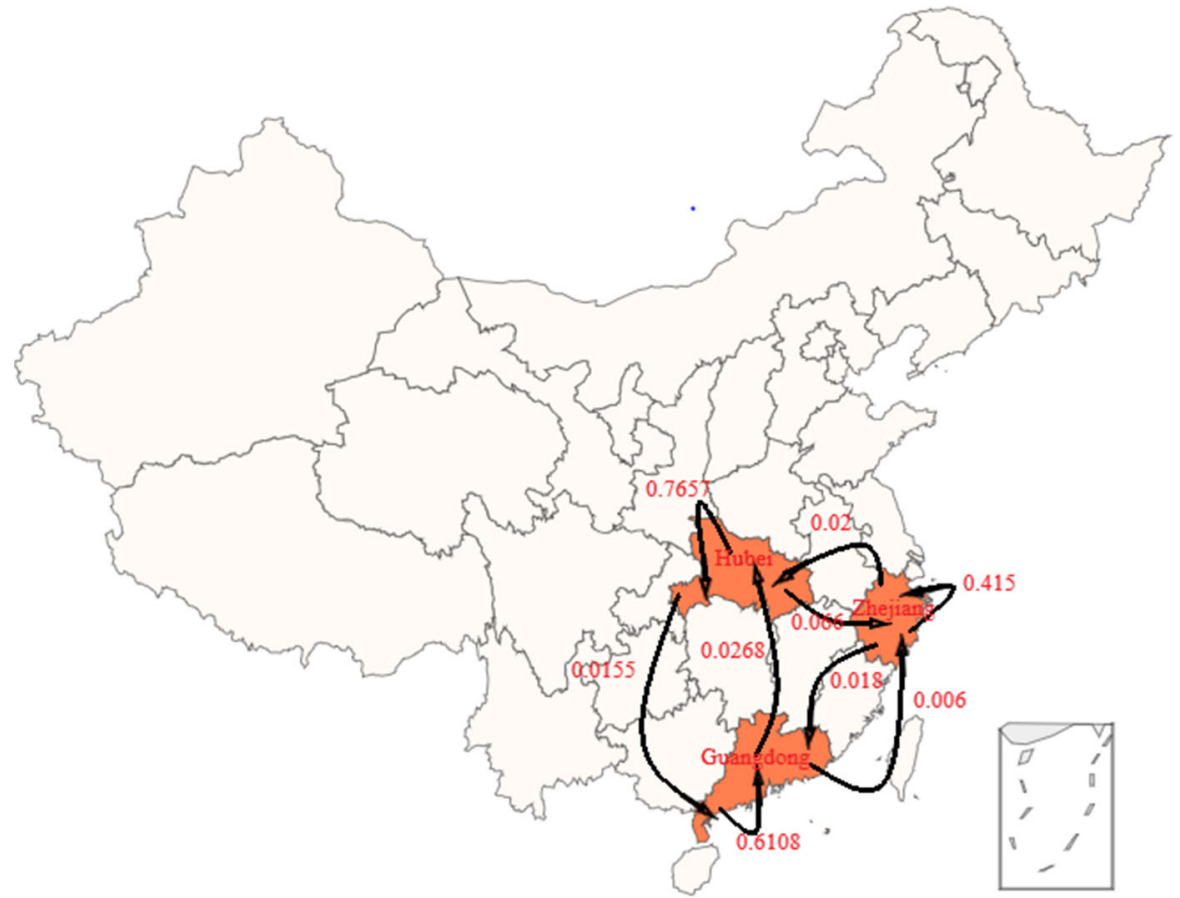

The matrix $A=\left(\alpha_{i j}\right)_{N \times N}$ is

$A=\left(\begin{array}{ccc}0.7657 & 0.0155 & 0.066 \\ 0.0268 & 0.6108 & 0.006 \\ 0.02 & 0.018 & 0.415\end{array}\right)$

One can see that the matrix $A$ is irreducible, and the directed graph $\mathscr{G}(A)$ is strongly connected. As pointed out in [3], the incubation period of the COVID-19 has been reported to be between 2 and 14 days. We take 5.2 days as time units which is the mean incubation period of the COVID-19 [29]. Hence, the rates of latent individuals becoming symptomatic (incubation rates) $\beta_{i}=$ $1 / 5.2, i=1,2,3$. One can obtain from the National Bureau of Statistics of China [30] that the annual death rate is equal to $7.13 \%$ (number of deaths/annual average number of population $\times 1000 \%$ ), and the annual birth rate is equal to $10.94 \%$ (number of births/average number of population $\times 1000 \%$ ). Hence, we can get that the death rate within 5.2 days is approximately equal to $7.13 \times 5.2 / 365 \% o=0.1016 \%$ o, and the birth rate within 5.2 days is approximately equal to $10.94 \times 5.2 / 365 \%$ o $=0.1559 \%$ o. We assume that the death rates of population occurring in $S_{i}, E_{i}, T_{i}, R_{i}$ compartments are equal to $0.1016 \%$, that is, $\mu_{i}^{S}=$ $\mu_{i}^{E}=\mu_{i}^{T}=\mu_{i}^{R}=0.1016 \% o, i=1,2,3$. Accord- ing to the data in [31], for COVID-19 the death rate of population occurring in $I_{i}$ compartment is $14 \%$. Hence, $\mu_{i}^{I}=14 \%, i=1,2,3$. We assume that the disease-related death rate of infective population who are not in treatment $\delta_{1 i}=14 \%, i=1,2,3$, and the transmission coefficient from infective population to treatment $\gamma_{i}=3 \%, i=1,2,3$. Since the diseaserelated death rate of infective population who are in treatment should be less than the death rate of population occurring in $I_{i}$ compartment, we assume that $\delta_{2 i}=9 \%, i=1,2,3$. In addition, there are lack of real data on the recovery rate of infective population, the fraction of total population who already have antibodies, and the proportion of recovery population who become susceptible again. Hence, we assume that $\sigma_{i}=10 \%, p_{i}=1 \%, \rho_{i}=1 \%, i=1,2,3$. We assume that the rates at which new individual enter the population of each province are equal to the birth rate, that is, $b_{i}=0.1559 \%, i=1,2,3$.

With above values of parameters, the matrix $M_{0}$ is

$M_{0}=\left(\begin{array}{lll}3.7386 & 0.0757 & 0.3222 \\ 0.1309 & 2.9823 & 0.0293 \\ 0.0977 & 0.0879 & 2.0263\end{array}\right)$.

Since $R_{0}=\rho\left(M_{0}\right)=3.7721>1$, one can know from Theorem 1 that the disease-free equilibrium $P_{0}$ 
Table 2 Daily reported cases in Hubei, Guangdong and Zhejiang provinces from January 23 to May 3

\begin{tabular}{llllllllll}
\hline Date & $1 / 23$ & $1 / 27$ & $1 / 31$ & $2 / 4$ & $2 / 8$ & $2 / 12$ & $2 / 16$ & $2 / 20$ & $2 / 24$ \\
\hline Hubei & 549 & 2567 & 6738 & 15,679 & 24,881 & 43,455 & 49,847 & 49,156 & 43,369 \\
Guangdong & 51 & 184 & 511 & 838 & 994 & 956 & 845 & 664 & 535 \\
Zhejiang & 43 & 172 & 584 & 832 & 902 & 818 & 701 & 555 & 410 \\
Date & $2 / 28$ & $3 / 3$ & $3 / 7$ & $3 / 11$ & $3 / 15$ & $3 / 19$ & $3 / 23$ & $3 / 27$ & $3 / 31$ \\
Hubei & 34,715 & 25,905 & 19,710 & 14,427 & 9605 & 6287 & 4318 & 2526 & 1283 \\
Guangdong & 370 & 214 & 95 & 60 & 47 & 62 & 87 & 118 \\
Zhejiang & 217 & 105 & 45 & 5 & 17 & 15 & 18 & 25 \\
Date & $4 / 4$ & $4 / 8$ & $4 / 12$ & $4 / 16$ & $4 / 21$ & $4 / 25$ & $4 / 29$ & $5 / 3$ \\
Hubei & 648 & 401 & 244 & 129 & 97 & 12 & 0 & 0 \\
Guangdong & 129 & 108 & 93 & 90 & 63 & 36 & 23 & 11 \\
Zhejiang & 32 & 31 & 27 & 21 & 15 & 10 & 4 & 2 \\
\hline
\end{tabular}

is unstable. As pointed out in [32], the obtained basic reproduction number $R_{0}=3.7721$ is reasonable for COVID-19 from China and overseas.

Since on January 23, Wuhan began to implement aggressive measures and policies, such as the closure of the city, nucleic acid testing, mask wearing, we set January 23, 2020 as time zero. We assume that $\sigma=1.1$. Since other parts of the China have implemented the closure of the city from January 23, the number of people who stay susceptible in each province is similar to that of its resident population. Hence, there are $S_{1}(0)=$ 59.27 million in Hubei province, $S_{2}(0)=115.21$ million in Guangdong province, and $S_{3}(0)=58.50$ million in Zhejiang province. The number of people who stay exposed in each province is similar to that of its capital cities, that is, $E_{1}(0)=11.08$ million in Hubei province, $E_{2}(0)=15.30$ million in Guangdong province, and $E_{3}(0)=10.36$ million in Zhejiang province. Table 2 depicts the daily reported cases in Hubei, Guangdong and Zhejiang provinces from January 23 to May 3 (see National Health Commission of the People's Republic of China [33]). On January 23, one can obtain from Health Commission of Hubei province that the number of infected people in Hubei is $I_{1}(0)=549$, and the recovery population of Hubei is $R_{1}(0)=20$. On January 23, one can obtain from Health Commission of Guangdong province that the number of infected people in Guangdong is $I_{2}(0)=53$, and the recovery population of Guangdong is $R_{2}(0)=2$. On January 23, one can obtain from Health Commission of Zhejiang province that the number of infected people in Zhejiang is $I_{3}(0)=43$, and the recovery population of Zhejiang is $R_{3}(0)=1$.

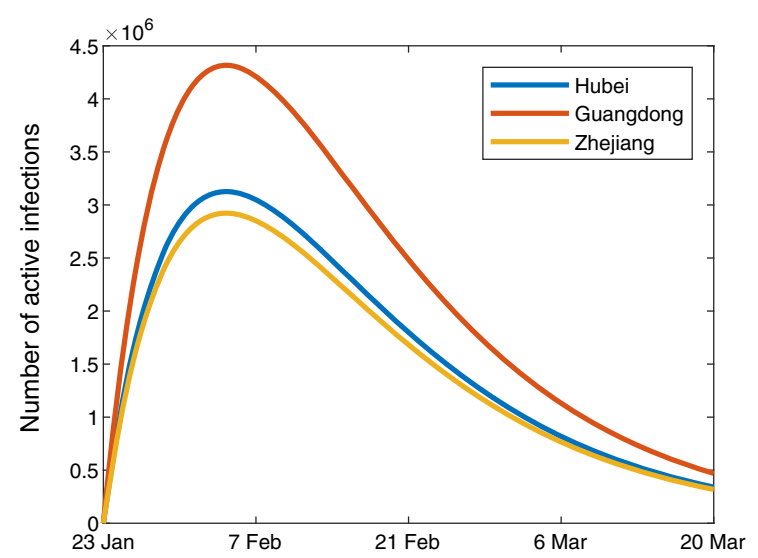

Fig. 3 The number of active infections in Hubei, Guangdong and Zhejiang provinces under no control

Based on the above data, we give the simulation of the COVID-19 outbreak in Hubei, Guangdong and Zhejiang provinces. Based on the model (1), Fig. 3 depicts the number of active infections in the three provinces under no control. One can see from Fig. 3 that the disease-free equilibrium is unstable and the number of COVID-19 infections in the three provinces will increase rapidly. Moreover, because Guangdong has a large population, its peak is higher than other provinces.

In order to make the disease-free equilibrium is globally asymptotically stable, we design two linear feedback controllers using Algorithm 1. By using Algorithm 1, we can obtain that the controlled set $G=$ $\{1,2,3\}$. If we choose $k_{1}^{I}=k_{1}^{E}=15, k_{2}^{I}=k_{2}^{E}=$ $30, k_{3}^{I}=k_{2}^{E}=30$, then the conditions of Theorem 

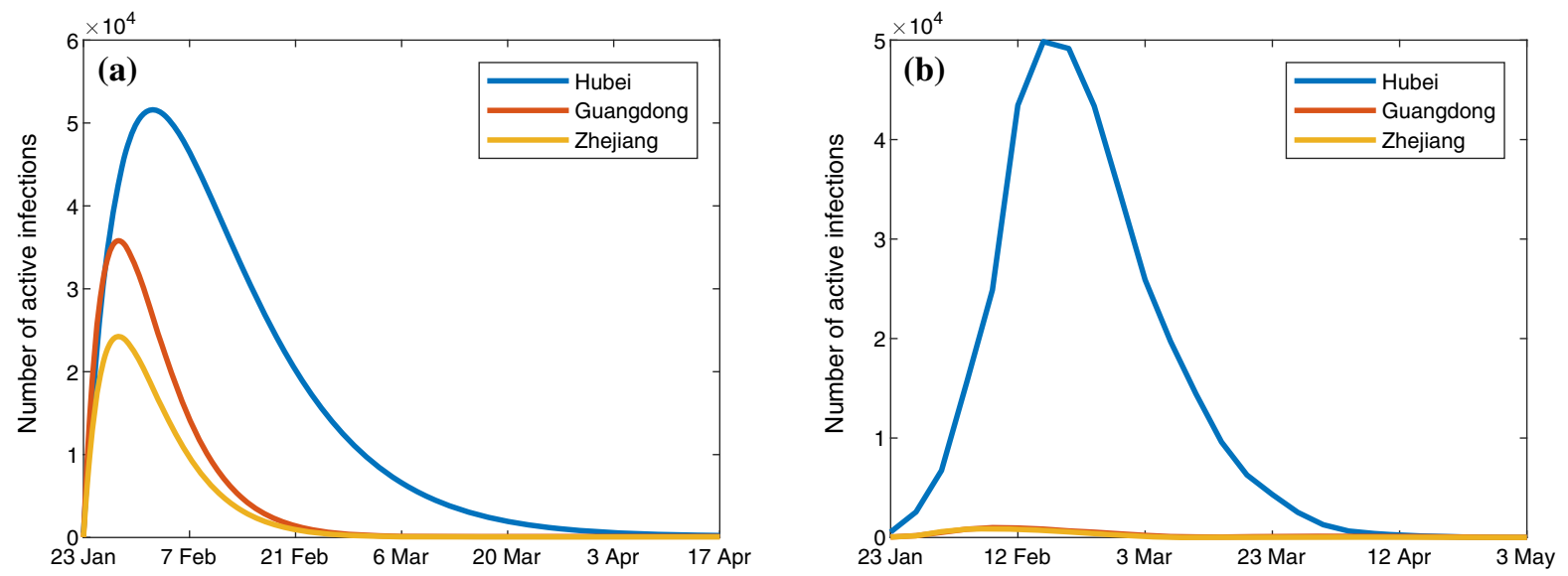

Fig. 4 a The number of active infections in Hubei, Guangdong and Zhejiang provinces under linear control; $\mathbf{b}$ The actual number of active infections in Hubei, Guangdong and Zhejiang provinces

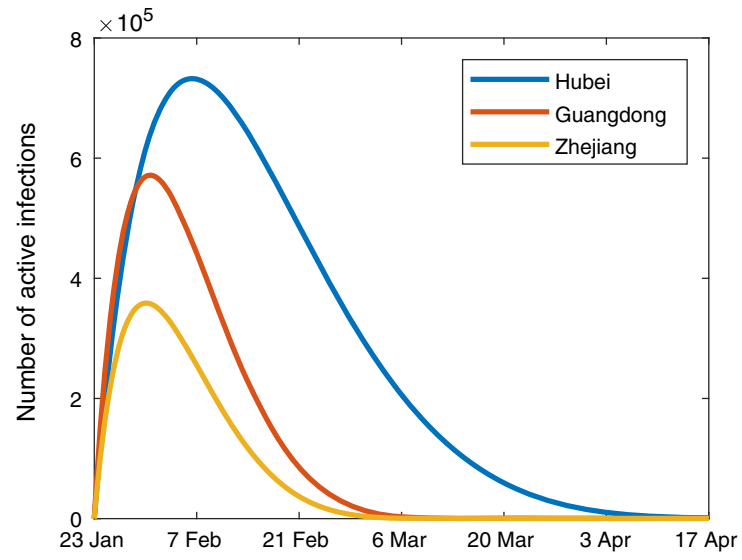

Fig. 5 The number of active infections in Hubei, Guangdong and Zhejiang provinces under finite time control

3 hold. One can see from Fig. 4a that the diseasefree equilibrium $P_{0}$ is globally asymptotically stable under proposed controllers. However, the number of active infections in Guangdong and Zhejiang provinces is much higher than the actual data (see Fig. 4b). The reason for this phenomenon is that we assume that there exists persistent mutual infections in three provinces. In fact, after the closure of Wuhan on January 23, the mutual infection between the three provinces should be very small. Hence, our simulation results can be regarded as the worst case that there are still many people moving among provinces.

If we design controllers as $\bar{u}_{i}^{E}\left(E_{i}\right)=\bar{k}_{i}^{E}\left(E_{i}\right)^{a}$, $\bar{u}_{i}^{I}\left(I_{i}\right)=\bar{k}_{i}^{I}\left(I_{i}\right)^{a}$, where $\bar{k}_{1}^{I}=\bar{k}_{1}^{E}=15, \bar{k}_{2}^{I}=\bar{k}_{2}^{E}=$ $30, \bar{k}_{3}^{I}=\bar{k}_{2}^{E}=30$ and $a=0.8$, then the conditions of Theorem 4 hold. One can see from Fig. 5 that the disease-free equilibrium is globally asymptotically stable under proposed controllers. However, these controllers do not significantly deduce the convergence time. Moreover, the peak number of active infections is much higher than linear control and actual data (see Fig. 4b). The reason for this phenomenon may be that the finite time controllers consume higher energy than linear control. This is a very meaningful phenomenon and we will solve it in our future work.

\section{Concluding remarks}

We have introduced a new multigroup COVID-19 model with immunity, and studied the stability of disease-free equilibrium and endemic equilibrium. When the basic reproduction number is less than or equal to one, and the infection graph is strongly connected, the disease-free equilibrium is globally asymptotically stable. When the disease-free equilibrium is unstable, some effective control strategies were given for the proposed multigroup COVID-19 model. The proposed control strategy contains two methods, one is to increase the number of treatment, and the other is to increase the detection to exclude the virus-free people. Future research will consider the control problem for the case that the infection graph infection graph is not strongly connected.

Acknowledgements The authors would like to thank the Editor, the Associate Editor and anonymous reviewers for their valu- 
able comments and suggestions that have helped to improve this paper considerably. This work was supported in part by the Natural Science Foundation of Chongqing of China under Grant cstc2019jcyj-msxmX0109, the Scientific and Technological Research Program of Chongqing Municipal Education Commission under Grant KJQN202000608.

Author contributions S. Zhai and J. Tao designed the research and wrote the manuscript. These two authors contributed equally to this work. H. Gao and G. Luo performed the data analysis. All authors read and approved the final manuscript.

\section{Compliance with ethical standards}

Conflict of interest The authors declare that they have no conflict of interest.

\section{References}

1. Chan, J.F.W., Yuan, S., Kok, K.H., To, K.K.W., Chu, H., Yang, J., Xing, F., Liu, J., Yip, C.C.Y., Poon, R.W.S., Tsoi, H.W., Lo, S.K.F., Chan, K.H., Poon, V.K.M., Chan, W.M., Ip, J.D., Cai, J.P., Cheng, V.C.C., Chen, H., Hui, C.K.M., Yuen, K.Y.: A familial cluster of pneumonia associated with the 2019 novel coronavirus indicating person-toperson transmission: a study of a family cluster. The Lancet 395(10223), 514-523 (2020)

2. Corman, V.M., Landt, O., Kaiser, M., Molenkamp, R., Meijer, A., Chu, D.K.W., Bleicker, T., Bruenink, S., Schneider, J., Schmidt, M.L., Mulders, D.G.J.C., Haagmans, B.L., van der Veer, B., van den Brink, S., Wijsman, L., Goderski, G., Romette, J.L., Ellis, J., Zambon, M., Peiris, M., Goossens, H., Reusken, C., Koopmans, M.P.G., Drosten, C.: Detection of 2019 novel coronavirus (2019-nCoV) by real-time RT-PCR. Eurosurveillance 25(3), 23-30 (2020)

3. Yang, Z., Zeng, Z., Wang, K., Wong, S.S., Liang, W., Zanin, M., Liu, P., Cao, X., Gao, Z., Mai, Z., Liang, J., Liu, X., Li, S., Li, Y., Ye, F., Guan, W., Yang, Y., Li, F., Luo, S., Xie, Y., Liu, B., Wang, Z., Zhang, S., Wang, Y., Zhong, N., He, J.: Modified SEIR and AI prediction of the epidemics trend of COVID-19 in China under public health interventions. J. Thorac. Dis. 12(3), 165-174 (2020)

4. Lajmanovich, A., Yorke, J.A.: A deterministic model for gonorrhea in a nonhomogeneous population. Math. Biosci. 28(3), 221-236 (1976)

5. Hethcote, H.W.: The mathematics of infectious diseases. SIAM Rev. 42(4), 599-653 (2000)

6. Huang, W., Cooke, K.L., Castillo-Chavez, C.: Stability and bifurcation for a multiple-group model for the dynamics of HIV/AIDS transmission. SIAM J. Appl. Math. 52(3), 835854 (1992)

7. Li, M.Y., Graef, J.R., Wang, L., Karsai, J.: Global dynamics of a SEIR model with varying total population size. Math. Biosci. 160(2), 191-213 (1999)

8. Li, M.Y., Shuai, Z.: Global-stability problem for coupled systems of differential equations on networks. J. Differ. Equ. 248(1), 1-20 (2010)
9. Sun, R.: Global stability of the endemic equilibrium of multigroup sir models with nonlinear incidence. Comput. Math. Appl. 60(8), 2286-2291 (2010)

10. Yuan, X., Wang, F., Xue, Y., Liu, M.: Global stability of an SIR model with differential infectivity on complex networks. Phys. A: Stat. Mech. Appl. 499, 443-456 (2018)

11. De la Sen, M., Ibeas, A., Alonso-Quesada, S.: On vaccination controls for the SEIR epidemic model. Commun. Nonlinear Sci. Numer. Simul. 17(6), 2637-2658 (2012)

12. Guo, L., Zhang, Y., Zheng, C.: Stabilization and optimal control of the SEITR epidemic model with vaccination. In: 2018 37th Chinese Control Conference (CCC), pp. 82258230 (2018)

13. Sharma, S., Samanta, G.P.: Stability analysis and optimal control of an epidemic model with vaccination. Int. J. Biomath. 08(3), 1550030 (2015)

14. Zhao, Z., Pang, L., Chen, Y.: Nonsynchronous bifurcation of SIRS epidemic model with birth pulse and pulse vaccination. Nonlinear Dyn. 79(4), 2371-2383 (2015)

15. Zhou, X., Cui, J.: Analysis of stability and bifurcation for an SEIV epidemic model with vaccination and nonlinear incidence rate. Nonlinear Dyn. 63(4), 639-653 (2011)

16. Blayneh, K., Cao, Y., Kwon, H.D.: Optimal control of vector-borne diseases: Treatment and prevention. Discret. Contin. Dyn. Syst.-Ser. B 11(3), 587-611 (2009)

17. Ranjit Kumar, U., Ashok Kumar, P., Sangeeta, K., Parimita, R.: Dynamics of an SEIR epidemic model with nonlinear incidence and treatment rates. Nonlinear Dyn. 96(4), 23512368 (2019)

18. Shen, M., Xiao, Y.: Global stability of a multi-group SVEIR epidemiological model with the vaccination age and infection age. Acta Appl. Math. 144(1), 137-157 (2016)

19. Wang, C., Fan, D., Xia, L., Yi, X.: Global stability for a multigroup SVIR model with age of vaccination. Int. J. Biomath. 11(5), 1850068 (2018)

20. Contreras, S., Andres Villavicencio, H., Medina-Ortiz, D., Biron-Lattes, J.P., Olivera-Nappa, A.: A multi-group SEIRA model for the spread of COVID-19 among heterogeneous populations. Chaos, Solitons \& Fractals 136, 109925 (2020)

21. Jean, D., Gabriel, T.: Heterogeneous social interactions and the COVID-19 lockdown outcome in a multi-group SEIR model. Math. Modell. Nat. Phenom. 15, 36 (2020)

22. Acemoglu, D., Chernozhukov, V., Werning, I., Whinston, M.D.: Optimal targeted lockdowns in a multi-group SIR model. National Bureau of Economic Research Working Paper 27102 (2020)

23. Huang, R., Liu, M., Ding, Y.: Spatial-temporal distribution of COVID-19 in China and its prediction: A data-driven modeling analysis. J. Infect. Dev. Ctries. 14(3), 246-253 (2020)

24. Xiao, D., Ruan, S.: Global analysis of an epidemic model with nonmonotone incidence rate. Math. Biosci. 208(2), 419-429 (2007)

25. Bhat, S.P., Bernstein, D.S.: Finite-time stability of continuous autonomous systems. SIAM J. Control Optim. 38(3), 751-766 (2000)

26. Hardy, G.H., Littlewood, J.E., Pólya, G.: Inequalities. Cambridge University Press, (1952)

27. van den Driessche, P., Watmough, J.: Further notes on the basic reproduction number. In: Brauer, F., van den Driessche, 
P., Wu, J. (eds.) Mathematical Epidemiology, pp. 159-178. Springer, Heidelberg (2008)

28. Sun, Y.Z., Leng, S.Y., Lai, Y.C., Grebogi, C., Lin, W.: Closed-loop control of complex networks: A trade-off between time and energy. Phys. Rev. Lett. 119(19), 198301 (2017)

29. Li, Q., Guan, X., Wu, P., Wang, X., Zhou, L., Tong, Y., Ren, R., Leung, K.S., Lau, E.H., Wong, J.Y., Xing, X., Xiang, N., Wu, Y., Li, C., Chen, Q., Li, D., Liu, T., Zhao, J., Liu, M., Tu, W., Chen, C., Jin, L., Yang, R., Wang, Q., Zhou, S., Wang, R., Liu, H., Luo, Y., Liu, Y., Shao, G., Li, H., Tao, Z., Yang, Y., Deng, Z., Liu, B., Ma, Z., Zhang, Y., Shi, G., Lam, T.T., Wu, J.T., Gao, G.F., Cowling, B.J., Yang, B., Leung, G.M., Feng, Z.: Early transmission dynamics in Wuhan, China, of novel coronavirus-infected pneumonia. New Engl. J. Med. 382(13), 1199-1207 (2020)

30. National Bureau of Statistics of China: Statistical communique of the people's republic of china on the 2019 national economic and social development. https://www.stats.gov. cn/english/PressRelease/202002/t20200228-1728917.html (2020)
31. Chen, A.T.C., Coura-Filho, G.B., Rehder, M.H.H.: Clinical characteristics of Covid-19 in China. New Engl. J. Med. 382(19), 1859-1860 (2020)

32. Liu, Y., Gayle, A., Wilder-Smith, A., Rocklov, J.: The reproductive number of COVID-19 is higher compared to SARS coronavirus. J. Travel Med. 27(2), taaa021 (2020)

33. National Health Commission of the People's Republic of China: Daily briefing on novel coronavirus cases in china. https://en.nhc.gov.cn/DailyBriefing.html (2020)

Publisher's Note Springer Nature remains neutral with regard to jurisdictional claims in published maps and institutional affiliations. 